\title{
Atomic-Level Fabrication of Crystalline Oxides in STEM
}

Q. He, ${ }^{1,2}$ S. Jesse, ${ }^{1,3}$ A.R. Lupini, ${ }^{1,2}$ D.N. Leonard, ${ }^{2}$ M.P. Oxley, ${ }^{4}$ O. Ovchinnikov, ${ }^{1,4}$ R. Unocic, ${ }^{1,3}$ A. Tselev, ${ }^{1,3}$ M. Fuentes-Cabrera ${ }^{3,6}$, B. G. Sumpter, ${ }^{1,3,6}$ S.J. Pennycook, ${ }^{5}$ S. V. Kalinin, ${ }^{1,3}$ and A.Y. Borisevich $^{1,2}$

${ }^{1}$ Institute for Functional Imaging of Materials, Oak Ridge National Laboratory, Oak Ridge, TN 37831

${ }^{2}$ Materials Sciences and Technology Division, Oak Ridge National Laboratory, Oak Ridge, TN 37831

${ }^{3}$ The Center for Nanophase Materials Science, Oak Ridge National Laboratory, Oak Ridge, TN 37831

${ }^{4}$ Dept of Physics and Astronomy, Vanderbilt University, Nashville, TN

${ }^{5}$ Dept of Materials Science and Engineering, The University of Tennessee Knoxville, Knoxville, TN

${ }^{6}$ Computer Science and Mathematics Division, Oak Ridge National Laboratory, Oak Ridge, TN 37831

Manipulation and control of the matter at the atomic level is one of the ultimate goals in nanoscience. The demonstration of atomic manipulation of xenon atoms by STM ${ }^{[1]}$ was one of the seminal achievements in this direction. Beyond the obvious impact of demonstrating the smallest artificially fabricated structure, this opened the possibilities for the fabrication of novel atomic structures including quantum corrals, ${ }^{[2]}$ standing electronic waves, ${ }^{[3]}$ atomic switches, ${ }^{[4]}$ molecular cascades for atom based computing, ${ }^{[5]}$ and quantum holographic devices. ${ }^{[6]}$ While the STM and non-contact AFM approaches are limited to material surfaces, an alternative paradigm for nano-patterning in bulk material is offered by electron beams. E-beam lithography in scanning electron microscope (SEM) geometry ${ }^{[7]}$ has been demonstrated to fabricate three dimensional structures at the nanometer scale. However atomic-level fabrication is only expected with highly energetic e-beams in (scanning) transmission electron microscope ((S)TEM). Recently in-situ fabrication of metallic nanowires of 2D $\mathrm{MoS}_{2}$ and $\mathrm{MoSe}_{2}$ materials have been demonstrated. However, there are very few reported studies of fabricating bulk material in (S)TEM. For several materials it has been shown that an amorphous area several tens of nm in size can be converted into a polycrystal ${ }^{[\underline{[}]}$ or single crystal. ${ }^{[9]}$ Recently, atomic rearrangements in amorphous materials have been reported. ${ }^{[10]}$ These observations suggest that (S)TEM beam can in principle be used to achieve sub-nanometer level bulk nanofabrication.

In this work, we demonstrate atomic-level sculpting of 3d crystalline oxide nanostructures from metastable amorphous precursor in a scanning transmission electron microscope (STEM). $\mathrm{SrTiO}_{3}$ nanowires can be fabricated epitaxially from the crystalline substrate following the beam path. This method can be used for producing crystalline structures as small as 1-2 $\mathrm{nm}$ and the process can be observed in situ with atomic resolution. Two interesting observations are made, as shown in Figure 1: Firstly, there is a certain threshold electron dose required, beyond which the crystallization is not observed. Secondly, the nucleation of the crystal preferably happens at the interface between the amorphous film and the crystalline substrate, and the new crystal grows epitaxially. Assuming an unphysically low thermal conductivity of the amorphous $\mathrm{SrTiO}_{3}$ material, Joule heat induced by electron beam was estimated to be at most $50 \mathrm{~K}$, which is still too low to be responsible for the phase transformation. The energy transfer is thus likely to be knock-on in nature. Atomistic molecular dynamics (MD) simulations verified the feasibility of beam-induced crystallization if the high-energy excitation is applied in the vicinity of the amorphous-crystalline interface.

We further demonstrate fabrication of arbitrary shape structures via control of the position and scan speed of the electron beam. Combined with broad availability of the atomic resolved electron microscopy platforms, these observations suggest the feasibility of large scale implementation of bulk 
atomic-level fabrication as a new enabling tool of nanoscience and technology, providing a bottom-up, atom-by-atom, complement to 3D printing. ${ }^{[11]}$

[1] D. M. Eigler, E. K. Schweizer, Nature 1990, 344, 524-526.

[2] M. F. Crommie, C. P. Lutz, D. M. Eigler, Science 1993, 262, 218-220.

[3] M. F. Crommie, C. P. Lutz, D. M. Eigler, Nature 1993, 363, 524-527.

[4] D. M. Eigler, C. P. Lutz, W. E. Rudge, Nature 1991, 352, 600-603.

[5] A. J. Heinrich, C. P. Lutz, J. A. Gupta, D. M. Eigler, Science 2002, 298, 1381-1387.

[6] C. R. Moon, C. P. Lutz, H. C. Manoharan, Nature Physics 2008, 4, 454-458.

[7] C. Vieu, F. Carcenac, A. Pepin, Y. Chen, M. Mejias, A. Lebib, L. Manin-Ferlazzo, L. Couraud, H. Launois, Applied Surface Science 2000, 164, 111-117.

[8] aI. Jencic, M. W. Bench, I. M. Robertson, M. A. Kirk, Journal of Applied Physics 1995, 78, 974-982; bA. Meldrum, L. A. Boatner, R. C. Ewing, Journal of Materials Research 1997, 12, 1816-1827.

[9] aY. Zhang, J. Lian, C. M. Wang, W. Jiang, R. C. Ewing, W. J. Weber, Physical Review B 2005, 72, 094112; bG. Zhu, G. A. Botton, in The 15th European Microscopy Congress, Manchester Central, United Kingdom, 2012.

[10] K. Zheng, C. Wang, Y.-Q. Cheng, Y. Yue, X. Han, Z. Zhang, Z. Shan, S. X. Mao, M. Ye, Y. Yin, E. Ma, Nat Commun 2010, 1, 24.

[11] The research is sponsored by the Division of Materials Sciences and Engineering, Office of Basic Energy Sciences, U.S. Department of Energy. BGS and MF-C were supported by the Center for Nanophase Materials Sciences which is sponsored at Oak Ridge National Laboratory by the Office of Science, Basic Energy Sciences, U.S. Department of Energy. Calculations made use of resources at the Oak Ridge Leadership Computing Facility at the Oak Ridge National Laboratory, which is supported by the Office of Science of the U.S. Department of Energy under Contract DE-AC05-00OR22725.

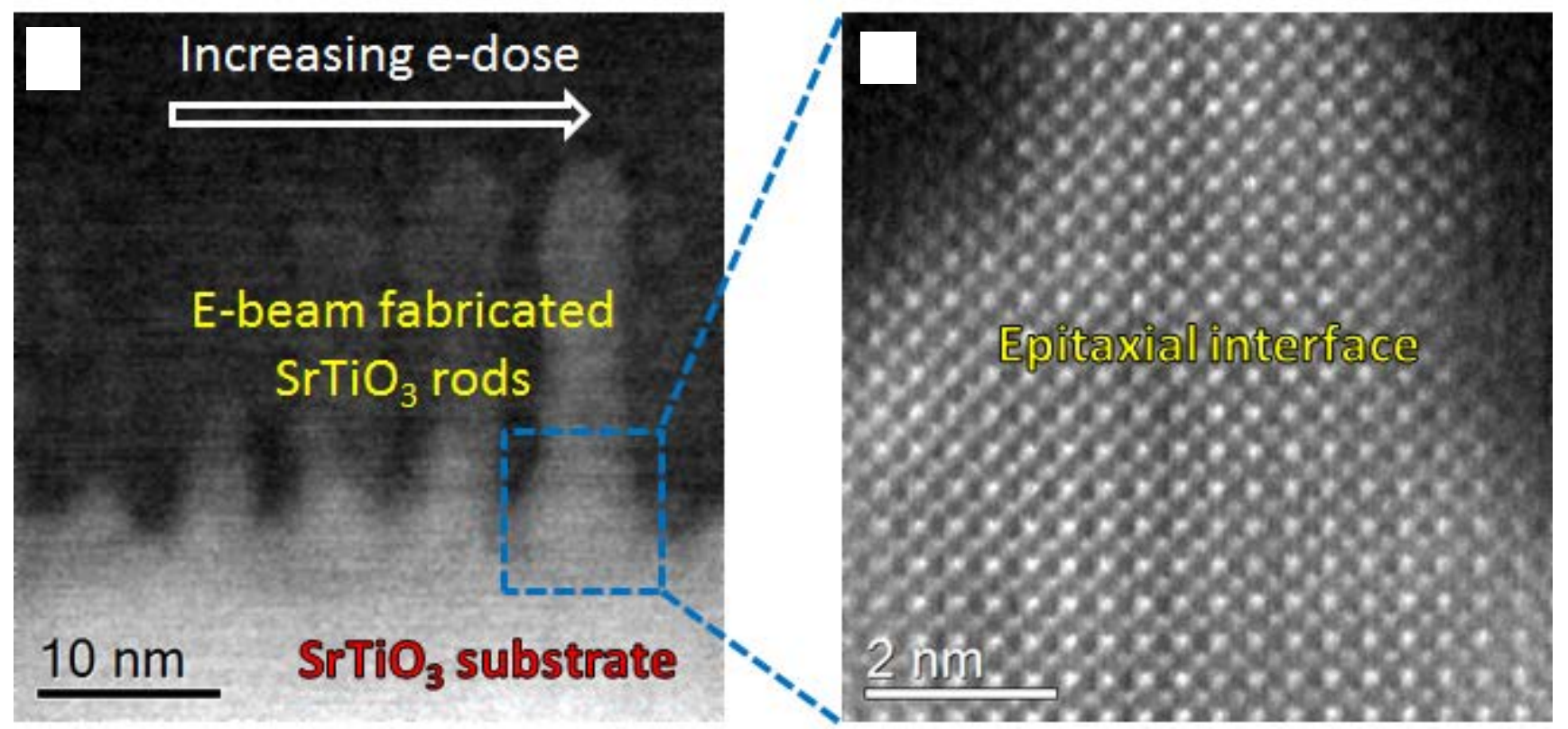

Figure 1. HAADF-STEM images of the e-beam fabricated epitaxial $\mathrm{SrTiO}_{3}$ rods. (a) A lower magnification HAADF image of a series of $\mathrm{SrTiO}_{3}$, demonstrating the effect of electron dose and the importance of the substrate. The formation of the SrTiO3 rods is more complete with higher electron dose. However for the left most rod, which was grown using the lowest electron dose, there is still growth near the interface. (b) A higher magnification HAADF image of the region highlighted in (a), showing the epitaxial character of the growth. 